\title{
Aspects of Diffraction at the Tevatron

\author{
Review and Phenomenological Interpretation of CDF Results on Diffraction
} \\ Presented at CIPANP-2003, New York City, 19-24 May 2003
}

\author{
Konstantin Goulianos \\ The Rockefeller University \\ 1230 York Avenue, New York, NY 10021 \\ (Experimental results are presented on behalf of the CDF Collaboration)
}

\begin{abstract}
Results on soft and hard diffraction obtained by the CDF Collaboration at the Fermilab Tevatron $\bar{p} p$ Collider are reviewed with emphasis on aspects of the data that point to the underlying QCD mechanism for diffraction. The results are interpreted in terms of a phenomenological approach in which diffraction is due to an exchange of low- $x$ partons subject to color constraints.
\end{abstract}

\section{INTRODUCTION}

Diffractive interactions between hadrons are characterized by the presence of one or more large rapidity gaps in an event. Processes which (do not) incorporate a hard partonic scattering in addition to the rapidity gap signature of diffraction are referred to as (soft) hard diffractive. A rapidity gap is a region of pseudorapidity ${ }^{1}$ devoid of particles. Rapidity gaps may be formed in non-diffractive (ND) interactions by multiplicity fluctuations. However, from Poisson statistics, the probability for a ND gap of width $\Delta \eta$ is expected to be $\mathrm{P}(\Delta \eta)=\exp [-\rho \Delta \eta]$, where $\rho$ is the average particle density per unit $\eta$. Thus, ND gaps are exponentially suppressed with increasing $\Delta \eta$. In contrast, diffractive gaps do not exhibit such a suppression. This aspect of diffraction could be explained if the exchange across the gap were a color singlet quark/gluon object with vacuum quantum numbers. For historical reasons, this object is referred to as Pomeron [1]. In this paper, we briefly review the results on soft and hard diffraction reported by the Collider Detector at Fermilab (CDF), present new results from Run II, and "interrogate" the data to learn about the partonic structure and factorization properties of Pomeron exchange. The information obtained is compared with expectations form the "renormalized gap probability" phenomenological model (RENORM), in which the Pomeron is formed from the underlying partonic structure of the interacting hadrons subject to the color-matching requirements appropriate for "vacuum exchange" [2, 3].

The paper is organized in two sections: soft diffraction and hard diffraction. For pedagogical reasons, experimental results and RENORM model expectations are presented concurrently and conclusions are interspersed within the main body of the presentation.

\footnotetext{
${ }^{1}$ We use rapidity and pseudorapidity interchangeably, since in the kinematic region of interest in this paper the pseudorapidity of a particle, defined as $\eta=-\ln \tan \frac{\theta}{2}$, where $\theta$ is the polar angle, is numerically very close to its rapidity, $y=\frac{1}{2} \frac{E+p_{L}}{E-p_{L}}$, where $p_{L}$ is the longitudinal momentum of the particle.
} 


\section{SOFT DIFFRACTION}

The following soft $\bar{p} p$ processes have been studied by CDF:


(ND)
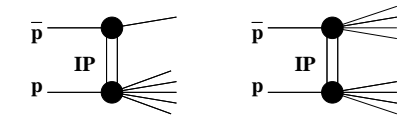

$\bar{p} p \rightarrow X$

$\bar{p} p \rightarrow \bar{p}+$ gap $+X$

$\bar{p} p \rightarrow X+$ gap $+Y$

DPE Double Pomeron Exchange [6]

$\bar{p} p \rightarrow \bar{p}+$ gap $+X+$ gap $+p$

$\bar{p} p \rightarrow \bar{p}+$ gap $+X+$ gap $+Y$

FIGURE 1. Diagrams and $\eta-\phi$ topologies of soft processes studied by CDF; the shaded areas are regions where particle production occurs and are referred to in this paper as diffractive clusters.

Diffraction has been traditionally treated phenomenologically in the framework of Regge theory. The connection of the theory to QCD is best seen by expressing cross sections in terms of rapidity gap and "diffractive cluster" variables, with the latter defined as regions of pseudorapidity where particle production occurs. The SDD process, for example, has two rapidity gaps and two diffractive clusters, which we designate, from left to right in Fig. 1] as $\Delta \eta_{1}, \Delta \eta_{1}^{\prime}, \Delta \eta_{2}$ and $\Delta \eta_{2}^{\prime}$. The gap $\Delta \eta_{1}$ can be thought of as being formed by the elastic scattering between the $\bar{p}$ and the cluster $\Delta \eta_{1}^{\prime}$, and the gap $\Delta \eta_{2}$ by the elastic scattering between the two diffractive clusters. Each gap is associated with a four-momentum transfer squared, $t$. There are 5 independent variables in SDD: the two rapidity gaps with their associated $t$-values and the center of the "floating" gap (non-adjacent to the $\bar{p}$ ), $\eta_{c}$. The Regge theory SDD differential cross section is given by

$$
\begin{array}{rc}
\frac{d^{5} \sigma}{d t_{1} d t_{2} d \Delta \eta_{1} d \Delta \eta_{2} d \eta_{c}}= & P_{\text {gap }}\left(t_{1}, t_{2}, \Delta \eta_{1}, \Delta \eta_{2}, \eta_{c}\right) \times \kappa^{2} \times \sigma_{t o t}\left(s^{\prime}\right) \\
P_{\text {gap }}\left(t_{1}, t_{2}, \Delta \eta_{1}, \Delta \eta_{2}, \eta_{c}\right)= & C \times F_{\bar{p}}^{2}\left(t_{1}\right) \times\left[e^{\left(\varepsilon+\alpha^{\prime} t_{1}\right) \Delta \eta_{1}}\right]^{2} \times\left[e^{\left(\varepsilon+\alpha^{\prime} t_{2}\right) \Delta \eta_{2}}\right]^{2} \\
\sigma_{\text {tot }}\left(s^{\prime}\right)= & \beta(0)^{2}\left(s^{\prime}\right)^{\varepsilon}=\beta(0)^{2} e^{\varepsilon \ln s^{\prime}}=\beta(0)^{2} e^{\varepsilon\left(\Delta \eta_{1}^{\prime}+\Delta \eta_{2}^{\prime}\right)}
\end{array}
$$

where $\beta(0)$ is the $\mathbb{P p}$ coupling at $t=0, \varepsilon$ and $\alpha^{\prime}$ the parameters of the Pomeron trajectory, $\alpha(t)=1+\varepsilon+\alpha^{\prime} t, \kappa=g^{I P I P I P} / \beta^{I P p}$ the ratio of the triple-Pomeron to the Pomeron-proton couplings, $s^{\prime}$ the diffractive cluster sub-energy defined by $\ln s^{\prime}=\Delta \eta^{\prime}=$ $\Delta \eta_{1}^{\prime}+\Delta \eta_{2}^{\prime}$, and $C$ a constant [3]. The parameter $\kappa$ has been measured to be $\kappa=$ $0.17 \pm 0.02$ [8].

The QCD connection. There are three factors in Eq. (1): $P_{g a p}, \kappa^{2}$ and $\sigma_{\text {tot }}$. Recalling that the total $\bar{p} p$ cross section is $\beta(0)^{2} e^{\varepsilon \ln s}$, the last factor is identified as the $\bar{p} p$ cross section at the diffractive sub-energy squared, $s^{\prime}$. From the optical theorem, the term $e^{\varepsilon \Delta \eta^{\prime}}$ is proportional to the forward elastic scattering amplitude at $s^{\prime}$. The fact that the 
two diffractive clusters are not contiguous does not present a conceptual problem in the parton model, in which the amplitude is $\sim e^{\varepsilon \Delta \eta_{i}^{\prime}}$ for each cluster [9] and thus the regions $\Delta \eta_{1}^{\prime}$ and $\Delta \eta_{2}^{\prime}$ add in the exponent. The full $t$-dependent parton model amplitude is:

$$
f_{\bar{p} p}(t, \Delta \eta) \propto e^{\left(\varepsilon+\alpha^{\prime} t\right) \Delta \eta} \quad \text { Parton Model Amplitude }
$$

Thus, in Eq. (2), the terms in the square brackets are identified as the amplitudes for elastic scattering between the diffractive clusters on either side of each gap, while $F_{\bar{p}}\left(t_{1}\right)$ is the $\bar{p}$ form factor. Finally, the parameter $\kappa$ is identified as the color factor required to produce a color singlet exchange; two such factors are needed in SDD, one for each gap.

Similar equations can be written for SD, DD and DPE [3]. In all cases the cross section factorizes into $P_{g a p}(\Delta \eta)$ and $\sigma_{t o t}\left(\Delta \eta^{\prime}\right)$ terms. The predicted shapes of the differential cross sections for all four processes agree with the CDF data [5, 6, 7, 8] . However, as seen in Fig. 2 $(\mathrm{a}, \mathrm{b})$, the $s$-dependence of the SD and DD cross sections is approximately flat at high energies, contrary to the Regge theory expectation of $\sim s^{2 \varepsilon}$. The culprit for this problem was identified [2] as the normalization of the $P_{g a p}(\Delta \eta)$ term, which is obtained from the elastic and total cross sections using factorization independently from the normalization of the $\sigma_{t o t}\left(\Delta \eta^{\prime}\right)$ term. Interpreting $P_{g a p}(\Delta \eta)$ as a gap probability distribution and renormalizing it to unity by dividing it by its integral over all phase space [2, 3] yields excellent agreement with the all data (see Fig. 2).



(a)


(c)

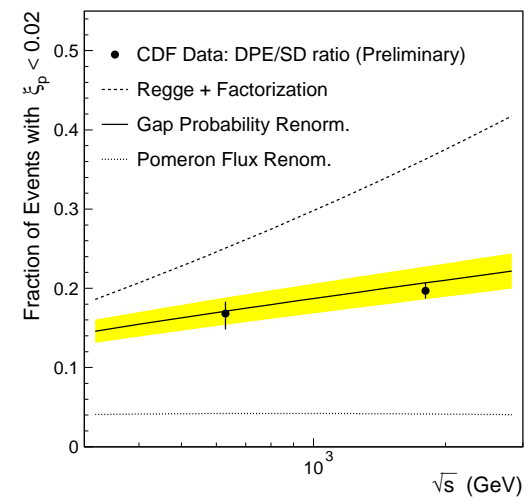

(d)

FIGURE 2. Soft diffraction cross sections compared with Regge theory and RENORM model predictions: (a) SD, (b) DD, (c) ratios of SDD to SD and DD to TOTAL, (d) ratio of DPE to SD. 


\section{HARD DIFFRACTION}

Hard diffraction processes studied by CDF include SD (dijet, $W, b$-quark and $J / \psi$ ), DD (dijet) and DPE (dijet) production, corresponding to the topologies shown in Fig. 3 .

(a)



$\phi$

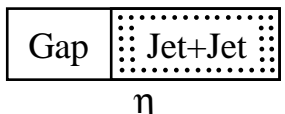

(b)

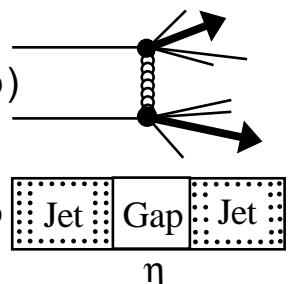

(c)

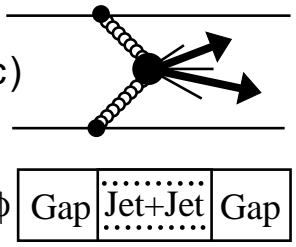

$\eta$

FIGURE 3. Topologies in $\eta-\phi$ space for hard (a) SD, (b) DD and (d) DPE processes.

Two types of results have been obtained: diffractive to non-diffractive cross section ratios (using the rapidity gap signature to select diffractive events), and diffractive to non-diffractive structure function ratios (using a Roman Pot Spectrometer to trigger on leading antiprotons). For a recent review of CDF Run I hard diffraction result see Ref. [10]. Here we summarize the aspects of the Run I results that point to the QCD structure of the Pomeron and present new results from Run II.

Run I rapidity gap results. (a) At $\sqrt{s}=1800 \mathrm{GeV}$, the SD/ND ratios for dijet, $W$, $b$-quark and $J / \psi$ production, as well the ratio of DD/ND dijet production, are all $\approx 1 \%$. This "gap fraction" is suppressed relative to QCD inspired theoretical expectations (e.g. 2 -gluon exchange) by a factor of $\sim 10$, which is comparable to the suppression factor observed in soft diffraction relative to Regge theory expectations based on factorization. (b) The gluon fraction of the diffractive exchange was determined from dijet, $W$ and $b$-quark production to be $0.54 \pm 0.15 \%$, which is similar to the ND fraction.

The above results indicate that (i) the diffractive structure function is similar to the ND one, apart from an overall suppression in normalization, and (ii) at fixed $\bar{p} p$ collision energy QCD factorization approximately holds within the diffractive sector.

Run I Roman Pot Results. (a) The diffractive structure function determined from SD dijet production is suppressed by a factor of $\sim 10$ relative to expectations based on extrapolations from parton densities determined from diffractive DIS at HERA. This suppression is approximately the same as that observed in soft diffraction. (b) The ratio of SD to ND structure functions behaves approximately as $x_{B j}^{-0.5}$. For a prediction of such behavior by the RENORM model see Ref. [11]. (c) The double-ratio of (DPE/SD)/(SD/ND) structure functions was found to be $5.3 \pm 2.0$, which is equal within errors to the ratio of (two-gap/one-gap)/(one-gap/no-gap) in soft diffraction (Fig. 3k).

Conclusions from Run I results. In both soft and hard diffraction processes cross sections factorize into two terms, one containing the cross section at the sub-energy of the diffractive cluster and another representing the gap probability distribution, which must be normalized to unity. A color factor is required for each gap. Diffraction appears as the interaction between low- $x$ partons subject to color-matching constraints imposed by the rapidity gap requirement, as prescribed by the RENORM model. 
Run II results. In Run II, diffractive data have been collected by CDF at $\sqrt{s}=1.96$ $\mathrm{TeV}$ and results obtained on the $Q^{2} \equiv\left(E_{T}^{j e t}\right)^{2}$ dependence of the diffractive structure function and on exclusive dijet production in hard DPE. Results are shown in Fig. 4.
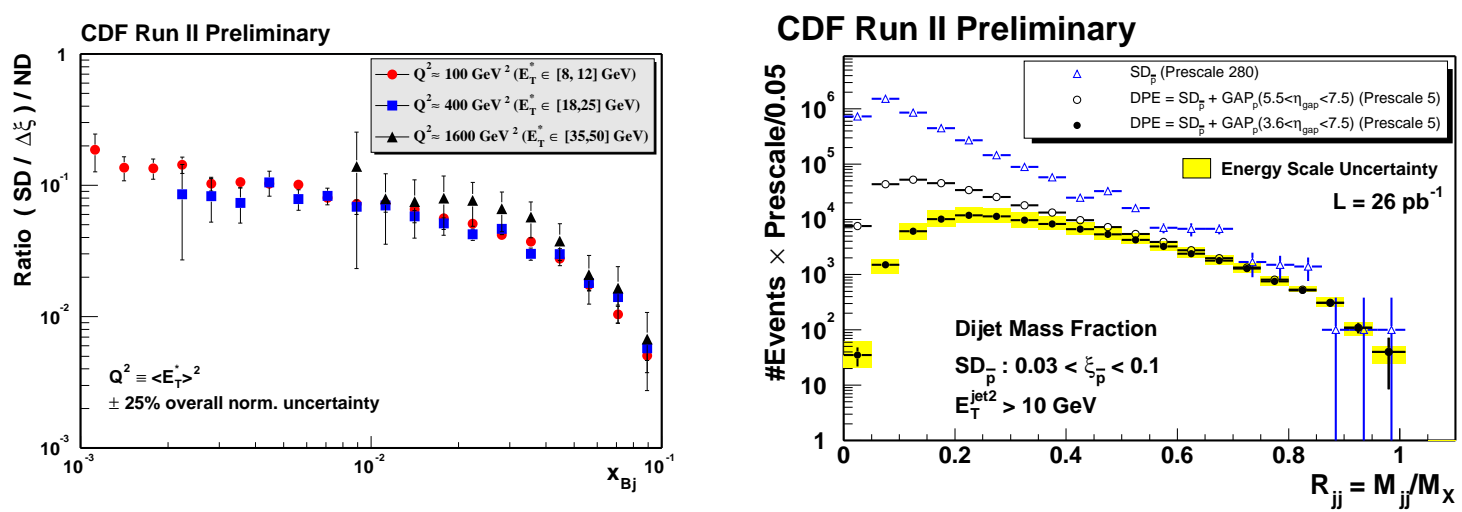

FIGURE 4. (left) Ratio of SD/ $\Delta \xi_{\bar{p}}$ over ND rates obtained from dijet data at various $Q^{2}$ ranges; (right) ratio of dijet mass to total mass "visible" in the calorimeters for dijet production in events with a leading antiproton within $0.3<\xi_{\bar{p}}<0.1$ and various gap requirements on the proton side: (triangles) no gap requirement, (open circles) gap in $5.5<\eta<7.5$, and (filled circles) gap in $3.5<\eta<7.5$.

The ratio of SD/ND rates, which in $\mathrm{LO} \mathrm{QCD}$ is equal to the ratio of the corresponding structure functions at a given $x_{B j}$, shows no appreciable $Q^{2}$ dependence. This result supports the RENORM model, in which the diffractive structure function is basically extracted from the non-diffractive one.

Exclusive dijet production in DPE, which has been proposed as a process on which to calibrate models of diffractive Higgs production [12], would appear in Fig. 4] as a peak in the vicinity of $R_{j j}=1$. No such peak is observed in the data. For dijets of minimum $E_{T}^{j e t}$ of $10 \mathrm{GeV}$ [25 GeV], the cross section for $R_{j j}>0.8$ is measured to be $970 \pm 65$ (stat) \pm 272 (syst) $[34 \pm 5 \pm 10] \mathrm{pb}$. Although similar values are obtained in Ref. [12] for exclusive dijets, we emphasize that no exclusive signal is seen in the data.

\section{REFERENCES}

1. V. Barone and E. Predazzi, High-Energy Particle Diffraction, Springer Press (2001).

2. K. Goulianos, Phys. Lett. B 358, 379 (1995); Erratum-ib. 363, 268 (1995).

3. K. Goulianos, "Diffraction in QCD," Presented at Corfu Summer Institute on Elementary Particle Physics (Corfu 2001), Corfu, Greece, 31 Aug - 20 Sep 2001; e-print Archive: hep-ph/0203141

4. F. Abe et al. (CDF Collaboration), Phys. Rev D 50, 5535 (1994).

5. T. Affolder et al. (CDF Collaboration), Phys. Rev Lett. 87, 141802 (2001).

6. D. Acosta et al. (CDF Collaboration), "Inclusive Double-Pomeron Exchange at the Fermilab Tevatron $\bar{p} p$ Collider," to be submitted to Phys. Rev. Letters.

7. D. Acosta et al. (CDF Collaboration), Phys. Rev Lett. 91, 011802 (2003).

8. K. Goulianos and J. Montanha, Phys. Rev. D 59, 114017 (1999).

9. E. Levin, "An Introduction to Pomerons," Preprint DESY 98-120.

10. K. Goulianos, "Diffraction at the Tevatron in Perspective," Presented at Workshop on Diffraction 2002, Alushta, Ukraine, 31 Aug - 6 Sep 2002; e-Print Archive: hep-ph/0306085

11. K. Goulianos, J.Phys.G26, 716 (2000).

12. V.A. Khoze, A.D. Martin and M.G. Ryskin, Eur. Phys. J. C23, 211 (2001); ib. C26, 229 (2002). 